\title{
Long term graft and recipient outcome of deceased donor renal transplantation at the National Kidney and Transplant Institute
}

\author{
Mark Javeson C. Tam ${ }^{1}$, Concesa B. Cabanayan-Casasola ${ }^{2}$ and Romina A.Danguilan*2 \\ ${ }^{1}$ Fellow-in-training, Department of Adult Nephrology, National Kidney and Transplant Institute, East Avenue, Quezon City, Philippines \\ ${ }^{2}$ Consultant, Department of Adult Nephrology, National Kidney and Transplant Institute, East Avenue, Quezon City, Philippines
}

\begin{abstract}
Background: The number of patients with end-stage renal disease (ESRD) is increasing and the gap between the demand for kidney transplantation (KT) and available donors is widening. Thus, deceased donation is very important to the donor pool for ESRD.

Objectives: This study aims to determine the long-term graft and recipient outcome of deceased donor renal transplantation at the NKTI from $2002-2007$ and to determine the donor and recipient factors that affect graft and recipient survival.

Materials and methods: This is a retrospective cohort of deceased donor KT from January 2002 to December 2007. Data were reviewed and collected from NKTI medical records and the Philippine Renal Disease Registry (PRDR). Recipient and donor demographic profile were expressed as frequency counts, percentages and means with standard deviation. Kaplan Meier was used to determine graft and patient survival and logistic regression to establish correlation between certain factors and survival

Results: Among 1,598 KT, 1488 were from living donors and 110 from deceased donors. 91 patients were included in this study. The mean recipient age was $40.40 \pm 11.8$ years and $65.9 \%$ were males. The primary renal diseases were chronic glomerulonephritis (63.7\%), diabetic nephropathy (18.7\%) and hypertensive nephrosclerosis (6.6\%). Around 39.6\% had 3 HLA mismatches and 61.5\% had at least 1 DR match. Majority received induction therapy (90.1\%) and 64.8\% had tacrolimus-based immunosuppressive regimen. The patient survival rate at 1, 3, 5 and 7 years was $91,89,86$ and 86 percent while graft survival was $89,79,73$ and 68 percent respectively. Infection was the leading cause of death. Cold Ischemia Time was significantly associated with patient survival $(\mathrm{P}=0.033)$ while patients with male donors had significantly better graft survival $(\mathrm{P}=0.001)$
\end{abstract}

Conclusion: There was an acceptable outcome of KT from deceased donors up to 7 years post KT.

\section{Introduction}

Kidney transplantation (KT) is the preferred treatment for end stage renal disease (ESRD). A successful transplant triples the life expectancy of a renal failure patient. The projected life expectancy with a transplant was 17.19 years compared with only 5.84 years on dialysis [1]. Hence, despite an initial higher risk of death, long-term survival for patients who underwent transplantation is significantly better compared with patients who remain on dialysis.

In addition, $\mathrm{KT}$ is more cost effective and improves quality of life. A study showed that the costs of patient therapy by hemodialysis is far greater than transplantation and its maintenance three and a half times costlier [2]. The difference in quality of life between dialysis and transplant patients is statistically significant with $18.12 \%$ greater in transplant patients. Another study showed that KT provides greater survival benefits to patients with end-stage renal disease, at less cost [3].

There is an increasing prevalence of ESRD and the demand for KT is increasing. Majority comprise living donation and unfortunately decease donation has not increased at an equivalent rate to meet the demand for KT [4]. In the Philippines, there are 10,000-12,000 new cases of ESRD annually [5]. 50- $60 \%$ will need KT but only $10 \%$ is done. Of those being transplanted, $90 \%$ from living donors and only $10 \%$ from deceased organ donation.
Various studies has been done in the Philippines regarding outcomes of deceased donor KT as shown in Table 1 [6-9]. These studies showed a remarkable improvement in the outcome of deceased donor KT.

\section{Objectives}

\section{General objectives}

1. To determine the long-term graft and recipient outcome of deceased donor renal transplantation at the National Kidney and Transplant Institute from 2002-2007

2. To determine the donor and recipient factors that affect graft and recipient survival

Correspondence to: Romina A. Danguilan, Chair of the Department of Adult Nephrology, National Kidney and Transplant Institute, East Avenue, Diliman, 1101 Quezon City, Philippines, Tel: 6329810300, Fax: 6329810368, E-mail: dr.radanguilan@gmail.com

Key words: Cadaveric donor kidney transplantation, graft survival, patient survival

Received: March 09, 2017; Accepted: April 05, 2017; Published: April 07, 2017 
Table 1. Studies on deceased donor kidney transplant in the Philippines.

\begin{tabular}{|c|c|c|c|c|c|c|}
\hline Author & $\mathbf{n}$ & KT Period & Immunosuppression & Induction Therapy & Patient Survival (\%) & Graft Survival (\%) \\
\hline Liquete [6] & 50 & $\begin{array}{c}1983- \\
1988\end{array}$ & $\begin{array}{c}\mathrm{CyA}+\text { pred } \\
\mathrm{CyA}+\mathrm{aza}+\text { pred }\end{array}$ & None & $\begin{array}{l}1 \text { year }-96 \\
3 \text { year }-81\end{array}$ & $\begin{array}{l}1 \text { year }-72 \\
5 \text { year }-50\end{array}$ \\
\hline Magcalas [7] & 228 & 1984- 1996 & $\begin{array}{c}\text { CyA+pred } \\
\text { CyA+aza+pred }\end{array}$ & none & $\begin{array}{c}1 \text { year }-81 \\
5 \text { year }-60 \\
10 \text { year }-48\end{array}$ & $\begin{array}{c}1 \text { year }-70 \\
5 \text { year }-42 \\
10 \text { year }-22\end{array}$ \\
\hline Ninalga [8] & 71 & $1995-2001$ & $\begin{array}{c}\text { CyA+aza+pred (46.5\%) } \\
\text { CyA+MMF+pred (46.5\%) } \\
\text { Tacro+aza+pred (2.8\%) } \\
\text { Tacro+MMF+pred (4.2\%) }\end{array}$ & $\begin{array}{c}\text { None }(84.5 \%) \\
\text { IL-2 blocker }(15.5 \%)\end{array}$ & $\begin{array}{l}1 \text { year }-80 \\
3 \text { year }-69\end{array}$ & $\begin{array}{l}1 \text { year }-65 \\
3 \text { year }-48\end{array}$ \\
\hline Overio [9] & 156 & $2007-2010$ & $\begin{array}{c}\text { Tacrolimus based } \\
(96.8 \%) \\
\text { CyA based } \\
(3.2 \%)\end{array}$ & $\begin{array}{c}\text { IL- } 2 \text { blocker } \\
(62.2 \%) \\
\text { Polyclonal antibody } \\
(37.8 \%)\end{array}$ & $\begin{array}{l}1 \text { year }-94 \\
3 \text { year }-90\end{array}$ & $\begin{array}{l}1 \text { year }-97 \\
3 \text { year }-96\end{array}$ \\
\hline
\end{tabular}

CyA: cyclosporine; pred: prednisone; aza: azathioprine; tacro: tacrolimus; MMF: mycophenolate mofetil

\section{Specific objectives}

1. To describe the recipients' demographic profile

2. To determine the incidence of graft and patient survival rate among recipients of deceased donor KT at 7 years post-KT

3. To describe the recipients' cause of mortality

4. To identify donor and recipient factors that significantly influences graft and patient survival at 7 years post KT

\section{Methodology}

\section{Research design}

Retrospective cohort study

\section{Study population}

All patients $\geq 18$ years of age who underwent primary deceased donor KT from January 2002 to December 2007 at the National Kidney and Transplant Institute were included in this study. Excluded were pediatric patients, foreigners and those who were lost to follow up for at least 12 months post KT.

\section{Materials and methods}

The following data were reviewed and collected from medical records and PRDR: a) recipients' demographic characteristics such as age, gender, cause of kidney disease, presence of diabetes mellitus, immunosuppressive regimen, induction therapy and immunologic status (Panel reactive antibody (PRA), number of HLA-ABDR mismatches and HLA-DR mismatches); b) donor factors such as age, gender and cold ischemia time (CIT)

Patients were followed up to 7 years post-KT to determine graft and patient survival.

\section{Definition of terms}

- Patient survival is the survival from the date of transplant until the date of death.

- Graft survival is the presence of renal function adequate to prevent the patient from resuming maintenance dialysis.

- Graft loss is patient's permanent return to dialysis.

\section{Statistical analysis}

The demographic profile of recipients and donors were expressed as frequency count, percentage and mean with standard deviation. Kaplan meier was used to determine graft and patient's survival rate. To establish correlation between certain factors and survival, logistic regression analysis was utilized.

\section{Ethical consideration}

Confidentiality of the subjects were maintained. Anonymity were ensured and each patient was assigned a case number.

\section{Results}

From January 2002 to December 2007, a total of 1,598 KT were performed at the NKTI, 1488 (93.1\%) were from living donors and $110(6.9 \%)$ were from deceased donors. Among the 110 recipients of deceased grafts, $91(82.7 \%)$ patients were included in this study and 19 (17.3\%) patients were excluded due to the following reasons: 3 subjects were foreigners; 10 pediatric patients and 6 subjects had incomplete data.

\section{Demographic characteristics}

The mean recipient age was $40.40 \pm 11.8$ years and $65.9 \%$ were males. The primary renal diseases were chronic glomerulonephritis $(63.7 \%)$, diabetic nephropathy (18.7\%) and hypertensive nephrosclerosis (6.6\%). Around $39.6 \%$ had 3 HLA mismatches and $61.5 \%$ with at least 1 DR match. Majority received induction therapy (90.1\%) and $64.8 \%$ had tacrolimus-based immunosuppressive regimen (Table 2).

\section{Graft and patient outcome}

The survival rate at $1,3,5$ and 7 years for patients was 91,89 , 86 and 86 percent while graft survival was $89,79,73$ and 68 percent respectively (Figure 1 and Figure 2). The leading cause of death was infection (76\%) followed by cardiovascular disease (14\%). Most of the death occurred less than 5 years post-KT (Table 3 ).

Factors associated with graft and patient survival at 7 years postkidney transplant

There were no recipient factors that was significantly associated with graft and patient survival at 7 years post-KT (Table 4 and Table 5).

Cold ischemia time was significantly associated with patient survival rate among the donor factors while the gender of the donor was significantly associated with graft survival rate. 


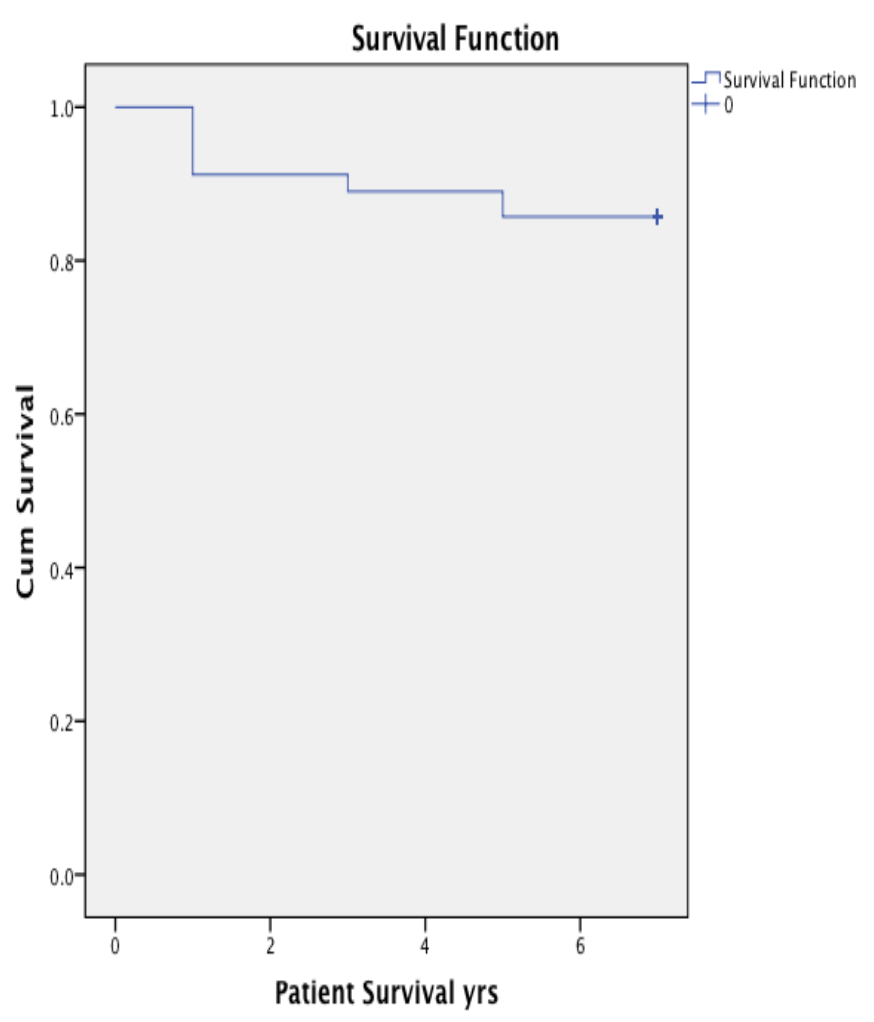

Figure 1. Kaplan Meier Patient survival rate.

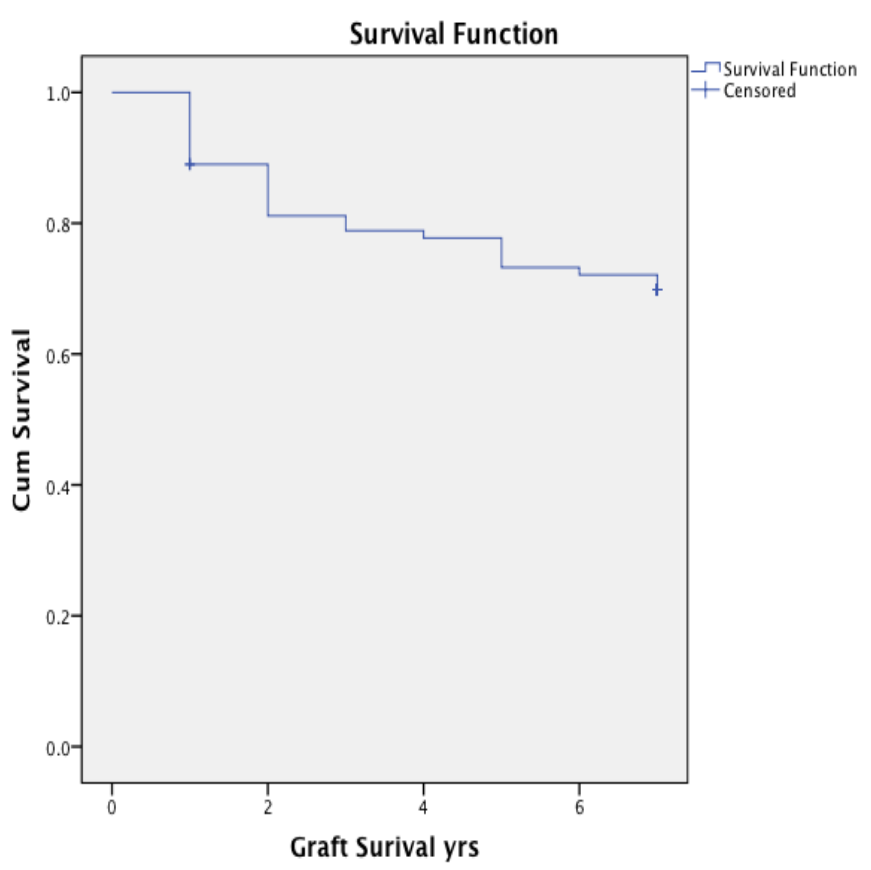

Figure 2. Kaplan Meier Graft survival rate.

The patient survival rate among patients was significantly different when grouped according to cold ischemia time. (Log Rank $=6.81$, $\mathrm{p}=0.033$ ). The cold ischemia time (CIT) with less than 12 had highest estimated mean survival rate (6.83 years), followed by $12-24$ CIT $(6.46$ years). Patients with CIT greater than 24 had the lowest estimated survival rate (5.52 years) (Table 6). Graft survival of patients was significantly different when grouped according to donor's gender $($ Log Rank $=11.055, \mathrm{p}=0.001)$. Patients who had male donors had significantly higher estimated survival rate (6.06 years) than patients with female donors (3.69 years) (Table 7).

\section{Discussion}

There has been continuous improvement in graft and patient survival from 1983 to 2010 [6-9] (Table 8).

Our study showed an improvement in graft survival when compared to studies done from 1983 to 2001 probably due to the improved immunosuppressive regimen, use of induction therapy and shorter CIT. When compared with the United States Renal Data System (USRDS) of 2013, our study was comparable in which their 1, 3 and 5 year patient survival rate were $94 \%, 86.6 \%$ and $75.5 \%$ while graft survival rates were $91.8 \%, 82.6 \%$ and $70.5 \%$ respectively [10].

Table 2. Demographic profile of recipients.

\begin{tabular}{|c|c|c|c|}
\hline \multicolumn{2}{|c|}{ Characteristics } & Frequency & Percentage \\
\hline \multirow{2}{*}{ Age (years) } & Less than 50 years old & 71 & 78.0 \\
\hline & 50 years old and above & 20 & 22.0 \\
\hline \multirow{2}{*}{ Gender } & Male & 60 & 65.9 \\
\hline & Female & 31 & 34.1 \\
\hline \multirow{2}{*}{$\begin{array}{c}\text { Presence of Diabetes } \\
\text { Mellitus }\end{array}$} & Yes & 14 & 15.4 \\
\hline & No & 77 & 84.6 \\
\hline \multirow{6}{*}{$\begin{array}{l}\text { Primary Renal } \\
\text { Disease }\end{array}$} & Chronic glomerulonephritis & 58 & 63.7 \\
\hline & Diabetic nephropathy & 17 & 18.7 \\
\hline & Hypertensive nephrosclerosis & 6 & 6.6 \\
\hline & Chronic pyelonephritis & 3 & 3.3 \\
\hline & $\begin{array}{l}\text { Autosomal dominant polycystic } \\
\text { kidney disease }\end{array}$ & 5 & 5.5 \\
\hline & Others & 2 & 2.2 \\
\hline \multirow{6}{*}{$\begin{array}{l}\text { Number of HLA- } \\
\text { ABDR Mismatch }\end{array}$} & 0 & 1 & 1.1 \\
\hline & 1 & 6 & 6.6 \\
\hline & 2 & 21 & 23.1 \\
\hline & 3 & 36 & 39.6 \\
\hline & 4 & 18 & 19.8 \\
\hline & 5 & 9 & 9.9 \\
\hline \multirow{3}{*}{$\begin{array}{l}\text { Number of DR } \\
\text { Matches }\end{array}$} & 0 & 24 & 26.4 \\
\hline & 1 & 56 & 61.5 \\
\hline & 2 & 11 & 12.1 \\
\hline \multirow{5}{*}{$\begin{array}{l}\text { Panel Reactive } \\
\text { Antibody (PRA) }\end{array}$} & Class $1>20 \%$ & 2 & 3.6 \\
\hline & $<20 \%$ & 54 & 96.4 \\
\hline & Class $2>20 \%$ & 0 & 0 \\
\hline & $<20 \%$ & 56 & 100 \\
\hline & No data & 35 & \\
\hline \multirow{3}{*}{$\begin{array}{c}\text { Immunosuppressive } \\
\text { Agents }\end{array}$} & Tacrolimus-based & 59 & 64.8 \\
\hline & CyA-based & 27 & 29.7 \\
\hline & Sirolimus- based & 5 & 5.5 \\
\hline \multirow{4}{*}{ Induction Therapy } & None & 9 & 9.9 \\
\hline & Basiliximab & 53 & 58.2 \\
\hline & Daclizumab & 5 & 5.5 \\
\hline & Alemtuzumab & 24 & 26.4 \\
\hline
\end{tabular}

Table 3. Causes of Death.

\begin{tabular}{|c|c|c|c|c|}
\hline \multirow[t]{2}{*}{ Cause of Death } & \multirow{2}{*}{$\begin{array}{l}\text { Frequency } \\
(\mathrm{n}=13)\end{array}$} & \multirow[t]{2}{*}{ Percentage } & \multicolumn{2}{|c|}{$\begin{array}{c}\text { Period of Death Post-KT } \\
\text { (years) }\end{array}$} \\
\hline & & & $<5$ & $\geq 5$ \\
\hline Infection Pneumonia (10) & 10 & 76.9 & 9 & 1 \\
\hline Cardiovascular & 2 & 15.4 & 1 & 1 \\
\hline Pulmonary Embolism & 1 & 7.7 & 1 & 0 \\
\hline
\end{tabular}


Tam MJC (2017) Long term graft and recipient outcome of deceased donor renal transplantation at the National Kidney and Transplant Institute

Table 4. Recipient Factors influencing 7-year patient survival.

\begin{tabular}{|c|c|c|c|c|c|}
\hline \multicolumn{6}{|c|}{ Patient Survival } \\
\hline Factors & No. of Events & Censored & $\begin{array}{c}\text { Estimated Mean Survival } \\
\text { (in years) }\end{array}$ & Log-rank Statistic Value & P-Value \\
\hline \multicolumn{6}{|c|}{ Recipient Age } \\
\hline Less than $50 \mathrm{y} / \mathrm{o}(\mathrm{n}=71)$ & 9 & 62 & 6.465 & 0.784 & 0.376 \\
\hline $50 \mathrm{y} / \mathrm{o}$ and above $(\mathrm{n}=20)$ & 4 & 16 & 5.8 & & \\
\hline \multicolumn{6}{|c|}{ Recipient Gender } \\
\hline Female $(\mathrm{n}=31)$ & 4 & 27 & 6.29 & 0.058 & 0.809 \\
\hline Male $(\mathrm{n}=60)$ & 9 & 51 & 6.333 & & \\
\hline \multicolumn{6}{|c|}{ Primary Renal Disease } \\
\hline $\begin{array}{c}\text { Glomerulonephritis } \\
(\mathrm{n}=58)\end{array}$ & 9 & 49 & \multirow{6}{*}{$\begin{array}{l}\text { No estimates were } \\
\text { computed because most } \\
\text { cases were censored. }\end{array}$} & \multirow{6}{*}{1.833} & \multirow{6}{*}{0.872} \\
\hline $\begin{array}{l}\text { Diabetic Nephropathy } \\
(\mathrm{n}=17)\end{array}$ & 3 & 14 & & & \\
\hline HPN $(n=6)$ & 1 & 5 & & & \\
\hline $\mathrm{CPN}(\mathrm{n}=3)$ & 0 & 3 & & & \\
\hline $\operatorname{APKD}(\mathrm{n}=5)$ & 0 & 5 & & & \\
\hline others $(\mathrm{n}=2)$ & 0 & 2 & & & \\
\hline \multicolumn{6}{|c|}{ DM } \\
\hline yes $(n=14)$ & 2 & 12 & 6.143 & 0.001 & 0.977 \\
\hline no(n=77) & 11 & 66 & 6.351 & & \\
\hline \multicolumn{6}{|c|}{ Induction } \\
\hline none $(\mathrm{n}=9)$ & 0 & 9 & $\begin{array}{l}\text { No estimates were } \\
\text { computed because most } \\
\text { cases were censored. }\end{array}$ & 1.582 & 0.208 \\
\hline with induction(n=82) & 13 & 69 & & & \\
\hline \multicolumn{6}{|c|}{ Immunosupression(ISA) } \\
\hline CYA base $(\mathrm{n}=27)$ & 6 & 21 & $\begin{array}{l}\text { No estimates were } \\
\text { computed because most } \\
\text { cases were censored. }\end{array}$ & 2.468 & 0.291 \\
\hline Tacrolimus base $(\mathrm{n}=59)$ & 7 & 52 & & & \\
\hline Sirolimus base $(\mathrm{n}=5)$ & 0 & 5 & & & \\
\hline \multicolumn{6}{|c|}{ PRA I } \\
\hline$<20 \%(\mathrm{n}=54)$ & 7 & 47 & $\begin{array}{l}\text { No estimates were } \\
\text { computed because most } \\
\text { cases were censored. }\end{array}$ & 0.283 & 0.595 \\
\hline$>20 \%(\mathrm{n}=2)$ & 0 & 2 & & & \\
\hline \multicolumn{6}{|c|}{ PRA II } \\
\hline $\begin{array}{l}<20 \%(\mathrm{n}=56) \\
>20 \%(\mathrm{n}=0)\end{array}$ & 7 & 49 & 6.393 & \multicolumn{2}{|c|}{$\begin{array}{l}\text { No comparison analysis was performed because the factor variable had only } \\
\text { one value for every stratum. }\end{array}$} \\
\hline \multicolumn{6}{|c|}{ DR Match } \\
\hline $0(\mathrm{n}=24)$ & 4 & 20 & 6.25 & 0.417 & 0.812 \\
\hline $1(\mathrm{n}=56)$ & 7 & 49 & 6.429 & & \\
\hline $2(\mathrm{n}=11)$ & 2 & 9 & 5.909 & & \\
\hline \multicolumn{6}{|c|}{$\mathrm{AB} M \mathrm{MM}$} \\
\hline $0(\mathrm{n}=1)$ & 0 & 1 & \multirow{6}{*}{$\begin{array}{l}\text { No estimates were } \\
\text { computed because most } \\
\text { cases were censored. }\end{array}$} & \multirow{6}{*}{4.328} & \multirow{6}{*}{0.503} \\
\hline $1(\mathrm{n}=6)$ & 1 & 5 & & & \\
\hline $2(\mathrm{n}=21)$ & 4 & 17 & & & \\
\hline $3(\mathrm{n}=36)$ & 7 & 29 & & & \\
\hline $4(\mathrm{n}=18)$ & 0 & 18 & & & \\
\hline $5(\mathrm{n}=9)$ & 1 & 8 & & & \\
\hline
\end{tabular}


Tam MJC (2017) Long term graft and recipient outcome of deceased donor renal transplantation at the National Kidney and Transplant Institute

Table 5. Recipient Factors influencing 7-year graft survival.

\begin{tabular}{|c|c|c|c|c|c|}
\hline \multicolumn{5}{|c|}{ Graft Survival } & \multirow[b]{2}{*}{ P-Value } \\
\hline Factors & No. of Events & Censored & $\begin{array}{l}\text { Estimated Mean Survival (in } \\
\text { years) }\end{array}$ & Log-rank Statistic Value & \\
\hline \multicolumn{6}{|c|}{ Recipient Age } \\
\hline Less than $50 \mathrm{y} / \mathrm{o}(\mathrm{n}=71)$ & 24 & 47 & 5.592 & 1.906 & 0.167 \\
\hline $50 \mathrm{y} / \mathrm{o}$ and above $(\mathrm{n}=20)$ & 3 & 17 & 6.231 & & \\
\hline \multicolumn{6}{|l|}{ Recipient Gender } \\
\hline Female $(\mathrm{n}=31)$ & 8 & 23 & 5.903 & 0.374 & 0.541 \\
\hline Male $(\mathrm{n}=60)$ & 19 & 41 & 5.621 & & \\
\hline \multicolumn{6}{|c|}{ PRD- Renal Disease } \\
\hline Glomerulonephritis(n=58) & 17 & 41 & 5.817 & 1.222 & 0.943 \\
\hline Diabetic Nephropathy(n=17) & 6 & 11 & 5.223 & & \\
\hline $\mathrm{HPN}(\mathrm{n}=6)$ & 1 & 5 & 6 & & \\
\hline $\mathrm{CPN}(\mathrm{n}=3)$ & 1 & 2 & 5.333 & & \\
\hline $\operatorname{APKD}(\mathrm{n}=5)$ & 1 & 4 & 5.8 & & \\
\hline Others $(\mathrm{n}=2)$ & 1 & 1 & 6.5 & & \\
\hline \multicolumn{6}{|c|}{$\mathrm{DM}$} \\
\hline Yes $(n=14)$ & 5 & 9 & 5.13 & 0.541 & 0.462 \\
\hline No(n=77) & 22 & 55 & 5.823 & & \\
\hline \multicolumn{6}{|c|}{ Induction } \\
\hline None $(\mathrm{n}=9)$ & 1 & 8 & 6.444 & 1.558 & 0.212 \\
\hline With induction $(\mathrm{n}=82)$ & 26 & 56 & 5.641 & & \\
\hline \multicolumn{6}{|c|}{ Immunosupression(ISA) } \\
\hline CYA base $(\mathrm{n}=27)$ & 9 & 18 & 5.453 & 0.488 & 0.783 \\
\hline Tacrolimus base $(\mathrm{n}=59)$ & 16 & 43 & 5.768 & & \\
\hline Sirolimus base $(\mathrm{n}=5)$ & 2 & 3 & 6.6 & & \\
\hline \multicolumn{6}{|c|}{ PRA I } \\
\hline$<20 \%(\mathrm{n}=54)$ & 18 & 36 & 5.613 & 0.05 & 0.824 \\
\hline$>20 \%(\mathrm{n}=2)$ & 1 & 1 & 7 & & \\
\hline \multicolumn{6}{|c|}{ PRA II } \\
\hline $\begin{array}{l}<20 \%(\mathrm{n}=56) \\
>20 \%(\mathrm{n}=0)\end{array}$ & 19 & 37 & 5.663 & $\begin{array}{l}\text { No comparison analysis is } \\
\text { variable has only one }\end{array}$ & $\begin{array}{l}\text { because the factor } \\
\text { very stratum. }\end{array}$ \\
\hline \multicolumn{6}{|c|}{ DR Match } \\
\hline $0(\mathrm{n}=24)$ & 6 & 18 & 6.009 & 0.553 & 0.758 \\
\hline $1(\mathrm{n}=56)$ & 17 & 39 & 5.665 & & \\
\hline $2(n=11)$ & 4 & 7 & 5.364 & & \\
\hline \multicolumn{6}{|c|}{$\mathrm{AB}$ MM } \\
\hline $0(\mathrm{n}=1)$ & 0 & 1 & \multirow{5}{*}{$\begin{array}{l}\text { No estimates are computed } \\
\text { because most cases are } \\
\text { censored. }\end{array}$} & 5.806 & 0.326 \\
\hline $1(\mathrm{n}=6)$ & 0 & 6 & & & \\
\hline $2(\mathrm{n}=21)$ & 7 & 14 & & & \\
\hline $3(\mathrm{n}=36)$ & 14 & 22 & & & \\
\hline $4(n=18)$ & 5 & 13 & & & \\
\hline $5(\mathrm{n}=9)$ & 1 & 8 & & & \\
\hline
\end{tabular}

Table 6. Donor factors Influencing 7-year patient survival.

\begin{tabular}{|c|c|c|c|c|c|}
\hline \multicolumn{6}{|c|}{ Patient Survival } \\
\hline Factors & No. of Events & Censored & $\begin{array}{c}\text { Estimated Mean Survival (in } \\
\text { years) }\end{array}$ & Log-rank Statistic Value & P-Value \\
\hline \multicolumn{6}{|c|}{ Donor Age } \\
\hline$\leq 20$ y.o $(\mathrm{n}=21)$ & 1 & 20 & 6.714 & \multirow{3}{*}{4.605} & \multirow{3}{*}{0.1} \\
\hline $21-40 \mathrm{y} / \mathrm{o}(\mathrm{n}=61)$ & 9 & 52 & 6.377 & & \\
\hline$\geq 41$ y.o $(\mathrm{n}=9)$ & 3 & 6 & 5 & & \\
\hline \multicolumn{6}{|c|}{ Donor Gender } \\
\hline Female $(n=13)$ & 3 & 10 & 5.615 & 1.086 & 0.297 \\
\hline Male $(\mathrm{n}=78)$ & 10 & 68 & 6.436 & & \\
\hline \multicolumn{6}{|c|}{ Cold Ischemia Time } \\
\hline$<12(\mathrm{n}=24)$ & 2 & 22 & 6.833 & \multirow{3}{*}{6.81} & \multirow{3}{*}{0.033} \\
\hline $12-24(\mathrm{n}=44)$ & 4 & 40 & 6.455 & & \\
\hline$>24(\mathrm{n}=23)$ & 7 & 16 & 5.522 & & \\
\hline
\end{tabular}


Table 7. Donor factors influencing 7-year graft outcome.

\begin{tabular}{|c|c|c|c|c|c|}
\hline \multicolumn{5}{|c|}{ Graft Survival } & \multirow[b]{2}{*}{ P-Value } \\
\hline Factors & No. of Events & Censored & $\begin{array}{c}\text { Estimated Mean Survival (in } \\
\text { years) }\end{array}$ & Log-rank Statistic Value & \\
\hline \multicolumn{6}{|c|}{ Donor Age } \\
\hline$\leq 20$ y.o $(\mathrm{n}=21)$ & 4 & 17 & 5.952 & \multirow{3}{*}{3.142} & \multirow{3}{*}{0.208} \\
\hline $21-40 \mathrm{y} / \mathrm{o}(\mathrm{n}=61)$ & 19 & 42 & 5.841 & & \\
\hline$\geq 41$ y.o $(\mathrm{n}=9)$ & 4 & 5 & 4.333 & & \\
\hline \multicolumn{6}{|c|}{ Donor Gender } \\
\hline Female $(n=13)$ & 8 & 5 & 3.692 & \multirow{2}{*}{11.055} & \multirow{2}{*}{0.001} \\
\hline Male $(\mathrm{n}=78)$ & 19 & 59 & 6.06 & & \\
\hline \multicolumn{6}{|c|}{ Cold Ischemia Time } \\
\hline$<12(\mathrm{n}=24)$ & 6 & 18 & 6.417 & \multirow{3}{*}{0.674} & \multirow{3}{*}{0.714} \\
\hline $12-24(\mathrm{n}=44)$ & 14 & 30 & 5.523 & & \\
\hline$>24(\mathrm{n}=23)$ & 7 & 16 & 5.348 & & \\
\hline
\end{tabular}

Table 8. Studies on graft and patient survival from 1983 to 2010.

\begin{tabular}{|c|c|c|c|c|c|c|}
\hline Author & $\mathrm{n}$ & KT Period & Immunosuppression & Induction Therapy & $\begin{array}{c}\text { Patient Survival } \\
(\%)\end{array}$ & $\begin{array}{c}\text { Graft Survival } \\
(\%)\end{array}$ \\
\hline Liquete [6] & 50 & $\begin{array}{c}1983- \\
1988\end{array}$ & $\begin{array}{c}\text { CyA+pred } \\
\text { CyA+aza+pred }\end{array}$ & None & $\begin{array}{l}1 \text { year }-96 \\
3 \text { year }-81\end{array}$ & $\begin{array}{l}1 \text { year }-72 \\
5 \text { year }-50\end{array}$ \\
\hline Magcalas [7] & 228 & 1984- 1996 & $\begin{array}{c}\mathrm{CyA}+\text { pred } \\
\text { CyA+aza+pred }\end{array}$ & None & $\begin{array}{c}1 \text { year }-81 \\
5 \text { year }-60 \\
10 \text { year }-48\end{array}$ & $\begin{array}{c}1 \text { year }-70 \\
5 \text { year }-42 \\
10 \text { year }-22\end{array}$ \\
\hline Ninalga [8] & 71 & $1995-2001$ & $\begin{array}{c}\text { CyA+aza+pred }(46.5 \%) \\
\text { CyA+MMF+pred }(46.5 \%) \\
\text { Tacro+aza+pred }(2.8 \%) \\
\text { Tacro+MMF+pred }(4.2 \%)\end{array}$ & $\begin{array}{c}\text { None }(84.5 \%) \\
\text { IL-2 blocker }(15.5 \%)\end{array}$ & $\begin{array}{l}1 \text { year }-80 \\
3 \text { year }-69\end{array}$ & $\begin{array}{l}1 \text { year }-65 \\
3 \text { year }-48\end{array}$ \\
\hline Tam & 91 & $2002-2007$ & $\begin{array}{c}\text { Tacrolimus based }(64.8 \%) \\
\text { CyA based }(29.7 \%) \\
\text { Sirolimus based }(5.5 \%)\end{array}$ & $\begin{array}{c}\text { None }(9.9 \%) \\
\text { IL-2 blocker }(58.2 \%) \\
\text { Monoclonal antibody } \\
\quad(31.9 \%)\end{array}$ & $\begin{array}{l}1 \text { year }-91 \\
3 \text { year }-89 \\
5 \text { year }-86 \\
7 \text { year }-86\end{array}$ & $\begin{array}{l}1 \text { year }-89 \\
3 \text { year }-79 \\
5 \text { year }-73 \\
7 \text { year }-68\end{array}$ \\
\hline Overio [9] & 156 & $2007-2010$ & $\begin{array}{c}\text { Tacrolimus based } \\
(96.8 \%) \\
\text { CyA based } \\
(3.2 \%)\end{array}$ & $\begin{array}{c}\text { IL- } 2 \text { blocker } \\
(62.2 \%) \\
\text { Polyclonal antibody } \\
(37.8 \%)\end{array}$ & $\begin{array}{l}1 \text { year }-94 \\
3 \text { year }-90\end{array}$ & $\begin{array}{l}1 \text { year }-97 \\
3 \text { year }-96\end{array}$ \\
\hline
\end{tabular}

CyA: cyclosporine; pred: prednisone; aza: azathioprine; tacro: tacrolimus; MMF: mycophenolate mofetil

Infection (76.9\%) was the most common cause of death in our study followed by cardiovascular disease (15.4\%). Compared to the United States Renal Data System (USRDS) wherein the most common cause of death is cardiovascular disease (31\%) followed by infection (19\%) and malignancies (10\%) [10]. However, in a study in India of 160 deceased donor KT between 2006-2009, the most common cause of death was infection which was comparable to our study [11]. Factors for the high incidence of infections were unhygienic conditions, late presentation and diagnosis, high cost of life-saving antimicrobial agents and lack of sensitive and specific diagnostic tools that were either not available or were too expensive $[11,12]$.

Among the recipient and donor factors studied, the donor's gender was noted to affect the 7-year graft survival in which male donor had better outcome than female $(\mathrm{p}$ value $=0.001)$. This was similar to the study involving 464 renal transplant centers in Europe in which both patient and graft survival were worse with a female donor. Graft survival in female recipients of male donors was $48.4 \pm 0.4$ year vs. $46.9 \pm 0.6$ year for female donors ( $p$ value 0.0020 ). In male recipients, actuarial survival was $46.5 \pm 0.3$ year for male donors vs. $42.1 \pm 0.5$ year for female donors ( $p$ value $<0.0001$ ). The assumption about the relative benefit of a male donor is that male kidneys have greater nephron number [13].

Patients with CIT of 12 hours had a significant patient survival. This is explained because there was less rejection with shorter CIT hence no need to give solumedrol pulsing hence no infection which is the main cause of patient mortality. The acute rejection with prolonged ischemia time could be reversed with solumedrol pulsing but could cause severe infection which may lead to death. United Network for Organ Sharing (UNOS) data noted a reduction in CIT during the 10year period (1990-2000) with an overall reduction of 4.8 hour and noted improvement in 3-year graft survival ( $80 \%$ in $1996-2000$ vs. $72 \%$ in 1990-1995 ( $\mathrm{p}<0.001$ ) [14] Another study showed that CIT had a significant effect on the 6-year graft survival, a 10-hour increase in CIT was associated with a hazard risk ratio (HRR) of 1.20 for graft failure ( $\mathrm{p}<0.001)$ [15]. In our study, CIT $<12$ hours showed better 7 -year graft survival compared to CIT $12-24$ hours and $>24$ hours however it did not achieved statistical significance. In contrast, CIT influence on patient survival achieved statistical significance ( $P$ value 0.033 ). CIT with less than 12 hours had highest estimated mean survival rate (6.83 years), followed by $12-24$ hours CIT ( 6.46 years) and $>24$ hours CIT had the lowest estimated survival rate (5.52 years).

\section{Conclusion}

There was an acceptable outcome of KT from deceased donors up to 7 years post KT. The most common cause of death was infection followed by cardiovascular disease. Among the recipient and donor factors, the donor's gender had effect on the 7-year graft survival in which male deceased donor KT had better graft survival compared to females and CIT was significantly associated with patient survival rate with CIT of less than 12 hours having better survival compared to CIT 12-24 hours and $>24$ hours. 


\section{References}

1. Oniscu GC, Brown H, Forsythe JL (2005) Impact of cadaveric renal transplantation on survival in patients listed for transplantation. J Am Soc Nephrol 16: 1859-1865. [Crossref]

2. Perovic S, Jankovic S (2009) Renal Transplantation vs Hemodialysis: Costeffectiveness Analysis. Vojnosanitetski Pregled 66(8): 639-644

3. Kalo Z, Jaray J (2001) Economic Evaluation of Kidney Transplantation versus Hemodialysis in Patients with End-Stage Renal Disease in Hungary. Prog Transplant September 11(3):188-193.

4. Neylan JF, Sayegh MH, Coffman TM, Danovitch GM, Krensky AM, et al. (1999) The allocation of cadaver kidneys for transplantation in the United States: consensus and controversy. ASN Transplant Advisory Group. American Society of Nephrology. J Am Soc Nephrol 10: 2237-2243. [Crossref]

5. Philippine Renal Registry 2010 Annual Report. Department of Health.

6. Liquete R (1990) Long Term Outcome of Cadaveric Kidney Patients. Philippine Journal of Surgical Specialties 45(3): 99-101.

7. Magcalas W, Liquete R (1996) Long Term Outcome of Cadaver Kidney Recipients. National Kidney and Transplant Institute Proceedings 1: 1-8.
8. Ninalga HD, Danguilan R (2005) Graft and Recipient Outcome of Cadaveric Transplantation at the National Kidney and Transplant Institute from 1995-2001. National Kidney and Transplant Institute Proceedings 1: 63-70.

9. Overio Y, Parayno A (2013) Graft and Recipient Outcome of Cadaveric Renal Transplantation at NKTI from 2007 to 2010 Unites States Renal Data System (USRDS) Annual Data Report. Renal Transplantation Vol. 2 Chapter 7: 283-294.

10. Gumber MR, Kute VB (2011) Deceased Donor Organ Transplantation - A SingleCenter Experience. Indian J Nephrol 21(3): 182-185.

11. Jha V, Chugh S, Chugh KS (2000) Infections in Dialysis and Transplant Patients in Tropical Countries. Kidney Int 57 (Supp1 74): S585-S593

12. Zeier M, Döhler B, Opelz G, Ritz E (2002) The effect of donor gender on graft survival. J Am Soc Nephrol 13: 2570-2576. [Crossref]

13. Salahudeen AK, May W (2008) Reduction in cold ischemia time of renal allografts in the United States over the last decade. Transplant Proc 40: 1285-1289. [Crossref]

14. Salahudeen AK, Haider N, May W (2004) Cold ischemia and the reduced long-term survival of cadaveric renal allografts. Kidney Int 65: 713-718. [Crossref]

Copyright: $\odot 2017$ Tam MJC. This is an open-access article distributed under the terms of the Creative Commons Attribution License, which permits unrestricted use, distribution, and reproduction in any medium, provided the original author and source are credited. 